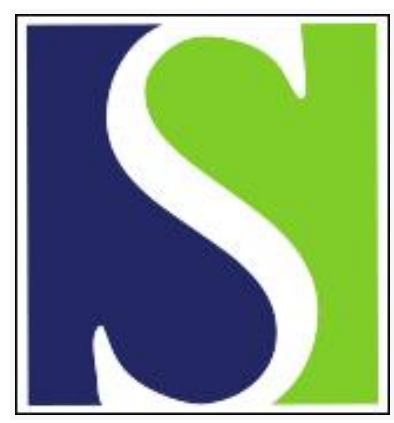

Scand J Work Environ Health 2015;41(4):356-367

https://doi.org/10.5271/sjweh.3497

Published online: 07 Apr 2015, Issue date: 01 Jul 2015

The ageing shift worker: a prospective cohort study on need for recovery, disability, and retirement intentions

by Gommans F, Jansen N, Stynen D, de Grip A, Kant I

Different shift work types are associated with adverse outcomes across the worker career. This study illustrates that shift work exposure duration and the healthy worker effect have an impact on these findings, probably resulting in an underestimation of the results. Measures to prevent adverse outcomes should be tailored for different types of shift work across the work career.

Affiliation: Department of Epidemiology, School CAPHRI, Maastricht University, P.O. Box 616, 6200 MD Maastricht, The Netherlands. Fleur.Gommans@maastrichtuniversity.nl

Refers to the following texts of the Journal: 1997;23(4):257-265 1999;25(2):85-99 2004;30(2):149-156 2006;32(3):232-240 2009;35(5):361-367 2011;37(5):402-410

The following article refers to this text: 2017;43(1):1-4

Key terms: ageing; ageing shift worker; cohort study; disability; healthy worker effect; longitudinal study; prospective cohort study; prospective study; recovery; retirement; retirement intention; shift work; shift worker; work schedule

This article in PubMed: www.ncbi.nlm.nih.gov/pubmed/25849304

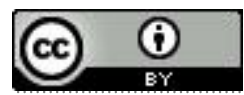




\title{
The ageing shift worker: a prospective cohort study on need for recovery, disability, and retirement intentions
}

\author{
by Fleur Gommans, MSC, ${ }^{1}$ Nicole Jansen, PhD, ${ }^{1}$ Dave Stynen, PhD, ${ }^{1}$ Andries de Grip, PhD, ${ }^{2}$ IJmert Kant, \\ $P h D^{1}$
}

Gommans F, Jansen N, Stynen D, de Grip A, Kant I. The ageing shift worker: a prospective cohort study on need for recovery, disability, and retirement intentions. Scand J Work Environ Health. 2015;41(4):356-367. doi:10.5271/sjweh.3497

Objectives This study investigates whether different shift work schedules, compared to day work, are associated with need for recovery (NFR), future disability, and retirement intentions for employees employed within different economic sectors over the course of their careers. Shift work exposure duration and the healthy worker effect are also examined.

Methods Data from the prospective Mastricht Cohort Study was used. Subsamples of industry (N=1877, all men) and healthcare (N=818, 624 women and 194 men) workers were separately investigated. GEE and Cox regression analyses were performed to investigate NFR longitudinally. Future disability was investigated using Cox regression, and retirement intentions were investigated using logistic regression analysis.

Results Compared to industry day workers, 3 -shift industry workers were at risk of becoming a case of elevated NFR during follow-up; 3- and 5-shift industry workers were at risk for future disability. In healthcare, irregular shift work was a risk factor for disability among older shift workers. No significant results were found regarding retirement intentions. Findings were probably an underestimation as exposure duration to shift work and the healthy worker effect affected the results.

Conclusions Shift work was associated with higher levels of NFR and a higher risk of disability. However, shift work is a multifaceted concept as different types of shift work schedules are differently associated with these outcomes. Different shift work types exist and shift work schedules allow for optimization, indicating that measures to prevent adverse outcomes should be tailored for different types of shift work and over the course of the work career.

Key terms healthy worker effect; longitudinal study; prospective study; shift work; work schedule.

In industrialized countries, a substantial proportion of the labor force consists of shift workers who work irregular or unusual hours compared to employees involved in normal day work schedules (1). In 2010, $17 \%$ of the workers in the European Union performed shift work, a number which has been increasing due to economic and technical reasons and the demand for a 24-hour society (2). Another ongoing trend is that older workers have to be maintained in the labor force (eg, mandatory retirement age postponement) due to ageing of the (work) population and the high relevance of sustainable employment for society, which is necessary to maintain current pension systems (3). Overall, this will result in an increase of older shift workers (4).

The performance of shift work however, can have adverse effects on employees' health and social life (5). Some common effects include sleep complaints, fatigue $(6,7)$, diseases and somatic health problems, e.g. cardiovascular diseases (8). Some studies suggest associations between shift work and depressive symptoms $(9,10)$. Moreover, social marginalization and psychosomatic disorders are common among shift workers (5). These effects can be explained by two major mechanisms: first, disruption of the circadian rhythm, which controls the

1 Department of Epidemiology, CAPHRI School for Public Health and Primary Care, Faculty of Health, Medicine and Life Sciences, Maastricht University, Maastricht, The Netherlands.

2 Research Centre for Education and the Labour Market (ROA), Maastricht University, Maastricht, The Netherlands.

Correspondence to: Fleur Gommans, Department of Epidemiology, School CAPHRI, Maastricht University, P.O. Box 616, 6200 MD Maastricht, The Netherlands. [E-mail: Fleur.Gommans@maastrichtuniversity.nl] 
daily physiology and behavior of humans, is related to many of these health effects. This rhythm aims to time functions such as sleep and performance so that they are optimal during the most suitable phase of the day. If this rhythm is frequently desynchronized due to shift work, this can result in health problems (11). Second, shift work might cause social disruption: many social activities are arranged according to the day-oriented rhythms of the general population (5), causing an imbalance between working times and social activities among shift workers.

To date, mixed findings (12) exist on whether these mechanisms result in more biological and social problems as shift workers age. For example, a study (13) concluded that shift workers become more prone to the chronobiological and social mechanisms at the age of 45-50, possibly resulting in the inability to continue performing shift work from this age on. However, few longitudinal studies have investigated the role of age on shift work tolerance (14). Therefore, to investigate whether the performance of shift work is associated with adverse outcomes that possibly vary across the lifespan, this study will focus on the concept of need for recovery (NFR) among shift workers. NFR reflects the short-term effects of a working day (15) and high levels of NFR are characterized by feelings of overload, irritability, and reduced performance (16). As NFR involves both the intensity of work-induced fatigue and the time-period required to return to a normal or pre-stressor level of functioning (17), it will be a valuable concept to investigate among shift workers. An earlier cross-sectional study (18) demonstrated that shift workers reported higher levels of NFR as compared to day workers; however this association has not yet been investigated over time. Furthermore, over time elevated levels of NFR can result in poor health and sickness absence (19). As a review (20) pointed out that the association between shift work and sick leave is schedule-specific, this study will also investigate the risk of shift workers to become unfit for work due to sickness or disability among different shift work types.

Also, retirement intentions will be a relevant outcome to investigate among shift workers, as a disruption of the social life could be associated with the decision to retire early, eg, work-to-family conflict can encourage workers to choose early retirement (21).

When investigating these outcomes among shift workers, several selection effects might have taken place and should therefore be investigated. The "healthy worker effect" is an expected selection effect. This effect is a type of bias in which less-healthy workers accumulate less occupational exposure because they, eg, retire earlier compared to healthier workers or switch to a job with lower exposure levels (22). Different manifestations of the healthy worker effect in terms of shift work are possible (23): a primary effect, in which employees simply never choose to engage in shift work, a secondary effect in which shift workers change their daily job routine after the occurrence of an event, or after experiencing that their current job does not match with their personal health or feelings and a tertiary selection effect, in which shift workers selectively drop-out from the study population. A review (12) concluded however, that the majority of longitudinal studies included in the review did not incorporate such effects.

Therefore, the present study aims to illustrate the possible manifestation of a secondary healthy worker effect. This is investigated by comparing shift workers who maintained a shift work job at an older age to those who moved from shift to day work, eg, in terms of NFR. Moreover, the shift worker's age will inevitably be related to the total exposure duration to shift work as older shift workers are more likely to have a longer total shift work exposure duration compared to younger workers (24) because shift work is often performed from a young age on.

Furthermore, the definition of shift work is ambiguous and shift work types may vary considerably, eg, in terms of shift duration and shift rotation direction. These different characteristics may involve different (health) effects for employees. For example, the number of working hours per week is often related to the type of shift: 3 -shift workers generally work $36-40$ hours per week whereas 5 -shift workers work on average 33 hours per week. A higher number of working hours per week was associated with a higher NFR (11). The present study therefore investigates whether different types of shift work schedules are differently associated with outcomes. Moreover, a sector-specific approach is valuable when investigating shift work, as different economic sectors imply differences in both the type of working time arrangements and the working conditions of employees. Therefore, different shift work types should be compared with day workers who are employed in the same sector. In this study, outcomes are investigated for two economic sectors separately. In conclusion, shift work is a broad concept and might be related to several adverse outcomes with a possibly varying impact across the work career.

This study aims to investigate the following research questions: (i) To what extent is shift work related to NFR and disability for employees in different age categories and involved in different work schedules, while taking into account possible confounders, investigated in two economic sectors separately? (ii) To what extent is shift work related to retirement intentions among older shift workers? (iii) Can a secondary healthy worker effect be illustrated in this study population? (iv) Is the exposure duration to shift work associated with differences in NFR? 


\section{Methods}

\section{Study population}

The study is based on data from the prospective Maastricht Cohort Study, which was set up in May 1998 and included 12140 participants from 45 different companies, employed in different jobs, sectors and trades. The study was conducted in accordance with the ethical standards laid down in the 1964 Declaration of Helsinki and its later amendments. Measurement T0 (May 1998) is the study baseline. The present study focused on shift workers from two economic sectors: industry and the healthcare sector. All analyses will be performed separately for the two economic sectors as different shift work schedules were investigated and different populations were selected.

To construct the study populations, first overall inclusion and exclusion criteria were formulated which were applied to both economic sectors. Before application of these criteria, the industry population comprised $\mathrm{N}=5862$ and the healthcare population comprised $\mathrm{N}=1825$ employees. Within each sector, two age categories were investigated: 35-44- and 45-60-year-olds (excluded: $\mathrm{N}=1742$ from industry, $\mathrm{N}=599$ from healthcare). Employees aged $\geq 45$ years were considered to be older employees, in accordance with the definition of the World Health Organization (WHO) (25). Employees involved in multiple jobs were excluded because only information on one job was available (excluded: $\mathrm{N}=142$ from industry, $\mathrm{N}=98$ from healthcare). Also, at T0 it was assessed whether employees had been on sick leave during the last four months. Employees who had been on sick leave during that period and had not (yet) resumed working, were excluded. Furthermore, employees who had been on sick leave during this four-month period and who, in the meantime, had resumed working, but were currently working under modified conditions related to former sick leave (which implies working for a limited number of hours per week and/or a restriction in work tasks) were excluded (excluded: $\mathrm{N}=366$ from industry, $\mathrm{N}=115$ from healthcare). In addition, different sector-specific inclusion- and exclusion criteria were applied within each economic sector to assemble the final populations.

Additional sector-specific criteria to construct the industry population were that all 3 - and 5-shift workers who performed night work were included. Industry day workers were included as a reference group (industry employees not involved in 3-shift, 5-shift work or day work were excluded: $\mathrm{N}=365$ ). Women were excluded as only a small number of women were employed in this sector ( $\mathrm{N}=213$ excluded). Also, employees working $<26$ hours per week were excluded as this was rather uncommon in this sector ( $\mathrm{N}=33$ excluded). Employees with a high educational level were excluded $(\mathrm{N}=1154$ excluded) as educational level was unevenly distributed among industry day and shift workers: the proportion of higher educated employees is larger among day workers and including them might result in a comparison between white-collar day workers and blue-collar shift workers, whereas the aim was to construct a homogenous study population. This resulted in a population of $\mathrm{N}=1877$ industry workers, comprising 1009 day workers, 354 3 -shift and 5145 -shift workers (of which $\mathrm{N}=1208$ and $\mathrm{N}=893$ were followed-up at $\mathrm{T} 8$ and $\mathrm{T} 9$ respectively). Additional sector-specific criteria to construct the healthcare population were that all irregular healthcare shift workers, irrespective of night work performance, were included. Performing night work was less common within healthcare $(44.8 \%$ of the irregular shift workers performed night work), resulting in a too small population if only night workers would be included. Healthcare day workers were included as a reference group (healthcare employees not involved in irregular shift work or day work were excluded: $\mathrm{N}=83$ ). Furthermore, employees working $<16$ hours per week were excluded ( $\mathrm{N}=101$ excluded) as this was uncommon in this sector. Pregnant women were excluded as they might have adjusted their work situation ( $\mathrm{N}=11$ excluded). The population of healthcare employees was $\mathrm{N}=818$, comprising of 577 day workers and 241 irregular shift workers (of which $\mathrm{N}=548$ and $\mathrm{N}=425$ were followed-up at $\mathrm{T} 8$ and $\mathrm{T} 9$ respectively).

\section{Measures}

Work schedules. To gain insight into the work schedules of employees at T0, a dichotomous question asked whether employees were currently engaged in day or shift work. Employees performing shift work were asked to indicate the specific type of shift in which they were engaged. In this study, 3-shift work implied a semicontinuous work schedule involving a 24-hour production from Monday through Friday carried out by three teams of employees, generally working 8-hour shifts. The majority of 3 -shift workers worked $\geq 36$ hours per week, while 5-shift work implied a full-continuous work schedule, spread over seven days including five altering teams, generally working 8-hour shifts. The majority of the 5-shift workers worked 26-35 hours per week. Irregular shift work implied working in frequently deviating work hours which can vary substantially every week. Next, the duration of being employed in that particular shift type was asked. Response options were $<1,1-5$, $6-10,11-20$, and $>20$ years and were recoded into three categories: short ( $\leq 10$ years), medium (11-20 years), and long ( $>20$ years) exposure duration to shift work.

Need for recovery. NFR was measured at T0 and at eight follow-up waves: T1 (September 1998), T2 (January 
1999), T3 (May 1999), T4 (September 1999), T5 (January 2000), T6 (May 2000), T7 (September 2000) and T8 (January 2001). A subscale from the Dutch Questionnaire on the Experience and Evaluation of Work (VBBA) (26) was used to assess NFR. This subscale contains 11 dichotomous items representing short-term effects of a working day and is recoded as such that a higher score indicates higher NFR. The recoded scores range from $0-100$. An earlier defined cut-off point of 6 on the 11-item scale was used to define cases with a low-medium (scoring below the cut-off point) and high (scoring above the cut-off point) NFR (27).

Future disability. As mentioned earlier, employees on sick leave at $\mathrm{T} 0$ were excluded from the analyses. To investigate disability during follow-up, employees first indicated whether they were currently employed or not. Those who were currently unemployed could indicate that they were retired (early), received sickness benefits, received disablement insurance, or were unemployed due to other reasons. Only those employees who indicated that they were currently unemployed and receiving sickness benefits or disablement insurance were classified as being disabled. The outcome was measured in nine follow-up waves: the first eight waves equal those of NFR (T1-T8) and were further extended with wave T9 (May 2002).

Retirement intentions. Retirement intentions were crosssectionally investigated at Maastricht Cohort Study wave October 2008 as this outcome was not measured in earlier waves of the cohort. As this outcome measure is mainly relevant for older employees, retirement intentions were only investigated among the 45-60 age category. One item (28) inventoried retirement intentions: "Have you considered retiring before the normal retirement age?" Response options were "no" "yes, sometimes" and "yes, often" and were recoded into two categories: "no" and "yes, sometimes" were recoded as having weak retirement intentions, "yes, often" was recoded as having strong retirement intentions.

Confounding factors. Potential confounders were measured at T0 and included several work, health, and personal determinants. To measure the psychological job demands of employees, a scale from the validated Dutch version (29) of the Job Content Questionnaire (JCQ) (30) was used. Two JCQ subscales were combined to measure decision latitude. All JCQ items had four response options, ranging from "strongly disagree" to "strongly agree". One dichotomous VBBA item measured emotional demands and one dichotomous item from the Dutch questionnaire on Work and Health (31) inventoried whether employees considered their job to be strenuous (yes/no). One dichotomous item deter- mined whether employees suffered from a long-term illness (yes/no). Two self-formulated items on the personal situation were included: whether employees took care of children living at home (yes/no) and whether employees took care of a chronically ill person at home (yes, partner/yes, child(ren)/yes, family/no). To dichotomize this item, response scores "yes, partner", "yes, child(ren)", and "yes, family" were grouped into "yes". Educational level was classified as low (primary school, lower vocational education), medium (lower secondary, intermediate vocational and upper secondary school), or high (upper vocational school, university). Gender and educational level were potential confounders corrected for only in the healthcare population, since the industry sector comprised only men and employees with a lowmedium educational level.

\section{Statistical analysis}

In all analyses, two economic sectors were separately investigated. Within each sector, results were stratified by type of shift work, and day workers from the same sector were included as a reference group. To describe the study population at baseline, $\chi^{2}$-tests tested for differences between shift and day workers in the prevalence of potential confounders and NFR caseness. Poisson regression analysis was conducted to investigate differences in mean NFR scores.

To investigate the continuous NFR scores longitudinally, generalized estimating equations (GEE, AR1 correlation structure) analyses were performed to account for correlated data arising from repeated measurements. An equal model (model 1) was applied for both economic sectors, partially adjusting for having a long-term illness, taking care of children living at home and taking care of a chronically ill person at home. Among those employed in the healthcare sector, also gender and educational level were included as potential confounders in this model. In the fully adjusted model (model 2), additionally adjustments for work environment determinants were included: psychological job demands, decision latitude, emotionally demanding and physically demanding work. Regression coefficients ( $\beta$ ) for the risk of a higher mean NFR score and standard errors (SE) were reported.

To investigate the influence of different shift work types on the dichotomous outcome measures NFR caseness and future disability, Cox regression analyses were performed. Time to first "NFR caseness" was modelled at T1, T2, T3, T4, T5, T6, T7, or T8 and time to first disability was additionally modelled at T9. For NFR caseness, models 1 and 2 were tested, including equal correction steps to the GEE models. The outcome future disability was not corrected for possible confounders, as it was hypothesized that overcorrection was likely to occur when disability was adjusted for potential 
confounders (eg, correction for having a long-term illness), as these confounders could constitute the cause of disability, which would possibly disturb the pathway linking work schedules and disability during follow-up. Hazard ratios (HR) and 95\% confidence intervals (95\% CI) were reported. Proportional hazard assumptions were met in all models. Logistic regression analysis tested for cross-sectional differences in retirement intentions between different types of shift work and day work.

The effect of the duration of exposure to shift work was investigated in the industry sector only (healthcare subsamples were too small for such analyses). Poisson regression analysis investigated the effect of duration of exposure to shift work, by comparing the mean NFR scores of industry shift workers with short, medium and long exposure duration at wave $\mathrm{T} 8$. Three age categories were investigated: $35-39,40-44$, and 45-50 years old. Here, more narrow age categories were selected to disentangle the relationship between exposure duration and age. By selecting narrower age categories, we aimed to ascribe differences in NFR to duration of exposure while aiming to rule out any possible age effects.

To investigate a possible healthy worker effect, time-lag GEE analyses (NFR at T-1 was investigated as a predictor of a transition from shift to day work at T) were performed, again only in the industry sector. A comparison of mean NFR scores was made between industry employees who continuously performed shift work throughout the follow-up period of 32 months and employees who altered from shift to day work at one point during the follow-up. As the number of transitions was relatively low, here all industry shift workers were included; irrespective of the type of shift work schedule (eg, also two-shift workers were included). Analyses were performed among shift workers aged $35-60$ years and shift workers aged $\geq 45$ years at T0 to investigate whether the healthy worker effect was also present when only older workers were investigated. In all analyses, a P-value of $<0.05$ was considered statistically significant. SPSS 20.0 (IBM, Armonk, NY, USA) was used to analyze the data.

\section{Results}

\section{Descriptives}

In table 1, baseline characteristics of the study population are shown. Differences between shift and day workers employed in the same sector were observed in the prevalence of work, health, and personal characteristics. For NFR caseness, the highest percentage of cases was found among 3-shift as compared to 5-shift and industry day workers. When stratifying for age, similar results were observed. Within healthcare, a higher percentage of NFR cases was found among irregular shift workers aged 45-60 years as compared to healthcare day workers, whereas among overall and 35-44 age categories no significant differences we revealed.

\section{Need for recovery}

In tables $2 \mathrm{a}$ and $2 \mathrm{~b}$, the continuous outcome scores of NFR are presented. In model 1 of the industry sector, NFR scores were significantly higher among 3- and 5 -shift workers as compared to industry day workers. Similar findings were observed in model 2. After age stratification, similar findings to the overall results were observed, except for 5-shift workers aged 45-60: NFR scores of these shift workers were not significantly higher as compared to day workers. In the healthcare sector, significant differences were found when comparing the overall NFR scores of irregular shift workers to day workers in model 1, whereas in model 2 no significant differences were observed. When stratifying for age, significantly higher NFR scores were observed among 45-60-year-old irregular workers as compared to day workers, in both models. As shown in table 3, among the industry sector, only 3 -shift work was found to be a risk factor for elevated NFR in model 1 as compared to industry day work. In model 2, this was no longer a risk factor. When stratifying for age, no significant results were demonstrated. Among healthcare employees, no statistically significant results were found.

\section{Future disability}

Both 3- and 5-shift work were found to be risk factors for future disability in the industry sector (table 4), also after age stratification. In healthcare, irregular shift work was only found to be a risk factor for future disability among 45-60 year olds.

\section{Retirement intentions}

To investigate the cross-sectional association between shift work and retirement intentions, data from Maastricht Cohort Study wave October 2008 was used. All 3-shift, 5-shift, and day workers aged 45-60 years employed in the industry sector were included, as applying other inclusion- and exclusion criteria would result in a too small population. The odd ratios (OR) regarding strong retirement intentions were generally in the expected direction, but failed to reach statistical significance: OR 1.45 (95\% CI 0.59-3.55) for 3-shift workers as compared to day workers and OR 1.34 (95\% CI 0.82 2.16) for 5-shift workers as compared to day workers. 
Table 1. Description of the work environment, health determinants, personal characteristics, and need for recovery (NFR) of the study population at baseline measurement (T0) (May 1998) according to work schedule in industry and the healthcare sector.

\begin{tabular}{|c|c|c|c|c|c|c|c|}
\hline & \multicolumn{4}{|c|}{ Industry ( $\mathrm{N}=1877$, all men) } & \multicolumn{3}{|c|}{ Healthcare ( $\mathrm{N}=818$, of which 624 were women) } \\
\hline & $\begin{array}{c}\text { Day work } \\
(\mathrm{N}=1009) \\
\%\end{array}$ & $\begin{array}{c}\begin{array}{c}\text { 3-shift } \\
(\mathrm{N}=354) \\
\%\end{array} \\
\end{array}$ & $\begin{array}{c}\begin{array}{c}5 \text {-shift } \\
(\mathrm{N}=514) \\
\%\end{array} \\
\end{array}$ & $P$-value & $\begin{array}{c}\text { Day work } \\
(\mathrm{N}=577) \\
\%\end{array}$ & $\begin{array}{c}\text { Irregular shift } \\
(\mathrm{N}=241) \\
\%\end{array}$ & P-value \\
\hline \multicolumn{8}{|l|}{ Work environment } \\
\hline Psychological job demands & & & & 0.004 & & & 0.765 \\
\hline Low & 32.4 & 30.3 & 39.4 & & 33.3 & 32.2 & \\
\hline Medium & 32.3 & 29.8 & 32.3 & & 33.3 & 36.0 & \\
\hline High & 35.4 & 39.9 & 28.3 & & 33.3 & 31.8 & \\
\hline Decision latitude & & & & $<0.0001$ & & & 0.006 \\
\hline Low & 25.8 & 53.2 & 49.8 & & 37.7 & 36.0 & \\
\hline Medium & 38.5 & 28.4 & 28.9 & & 32.0 & 42.7 & \\
\hline High & 35.8 & 18.4 & 21.3 & & 30.2 & 21.3 & \\
\hline Emotional demanding work & & & & $<0.0001$ & & & $<0.0001$ \\
\hline Yes & 20.4 & 33.3 & 26.6 & & 51.0 & 66.8 & \\
\hline No & 79.6 & 66.7 & 73.4 & & 49.0 & 33.2 & \\
\hline Physically demanding work & & & & $<0.0001$ & & & $<0.0001$ \\
\hline Yes & 16.3 & 40.6 & 34.9 & & 29.4 & 59.7 & \\
\hline No & 83.7 & 59.4 & 65.1 & & 70.6 & 40.3 & \\
\hline \multicolumn{8}{|l|}{ Health and lifestyle } \\
\hline Presence of a long-term illness & & & & 0.018 & & & 0.719 \\
\hline Yes & 25.6 & 27.6 & 19.9 & & 25.4 & 26.6 & \\
\hline No & 74.4 & 72.4 & 80.1 & & 74.6 & 73.4 & \\
\hline \multicolumn{8}{|l|}{ Personal characteristics } \\
\hline Educational level & & & & $<0.0001$ & & & $<0.0001$ \\
\hline Low & 24.8 & 63.0 & 49.4 & & 15.1 & 14.2 & \\
\hline Medium & 75.2 & 37.0 & 50.6 & & 43.4 & 71.6 & \\
\hline High & * & * & * & & 41.5 & 14.2 & \\
\hline $\begin{array}{l}\text { Taking care of children living at } \\
\text { home }\end{array}$ & & & & $<0.0001$ & & & 0.001 \\
\hline Yes & 56.7 & 66.8 & 66.5 & & 59.4 & 71.7 & \\
\hline No & 43.3 & 33.5 & 33.5 & & 40.6 & 28.3 & \\
\hline $\begin{array}{l}\text { Taking care of a chronically ill } \\
\text { person at home }\end{array}$ & & & & 0.969 & & & 0.550 \\
\hline Yes & 15.4 & 15.1 & 15.7 & & 19.1 & 20.9 & \\
\hline No & 84.6 & 84.9 & 84.3 & & 80.9 & 79.1 & \\
\hline \multicolumn{8}{|l|}{ Need for recovery } \\
\hline Cases of high NFR & & & & $<0.0001$ & & & 0.354 \\
\hline Yes & 20.1 & 34.9 & 26.3 & & 19.2 & 22.1 & \\
\hline No & 79.9 & 65.1 & 73.7 & & 80.8 & 77.9 & \\
\hline $\begin{array}{l}\text { Cases of high NFR in age category } \\
35-44 \text { years }\end{array}$ & & & & $<0.0001$ & & & 0.840 \\
\hline Yes & 16.2 & 32.9 & 25.5 & & 17.3 & 16.6 & \\
\hline No & 83.8 & 67.1 & 74.5 & & 82.7 & 83.4 & \\
\hline $\begin{array}{l}\text { Cases of high NFR in age category } \\
45-60 \text { years }\end{array}$ & & & & $<0.0001$ & & & 0.012 \\
\hline Yes & 22.0 & 39.4 & 27.4 & & 21.8 & 36.9 & \\
\hline No & 78.0 & 60.6 & 72.6 & & 78.2 & 63.1 & \\
\hline
\end{tabular}

${ }^{\star}$ Employees with a high educational level were excluded from the analyses.

\section{Exposure duration to shift work}

The duration of exposure to shift work was investigated in three age categories: $35-39,40-44$ and $45-50$ year old industry workers (table 5). In the youngest age category, a longer exposure duration was associated with lower NFR scores. In the 40-44 category, a longer duration was associated with higher NFR scores. Among 45-50 year olds, the highest NFR scores were found among those with medium exposure duration and the lowest scores among those with a long exposure duration to shift work.

\section{Healthy worker effect}

To illustrate a possible secondary healthy worker effect among both the total industry shift work population and among older industry shift workers, asso- 
Table 2a. Overall and age-stratified regression coefficients and standard errors for continuous need for recovery scores according to work schedule among the industry and healthcare sector (TO-T8) [SE=standard error.]

\begin{tabular}{|c|c|c|c|c|c|c|c|c|c|c|c|c|c|c|c|c|c|c|}
\hline \multirow[t]{3}{*}{ Industry } & \multicolumn{9}{|c|}{ Model $1^{\text {a }}$} & \multicolumn{9}{|c|}{ Model $2^{b}$} \\
\hline & \multicolumn{3}{|c|}{$\begin{array}{c}\text { Overall } \\
(\mathrm{N}=1791, \text { all men) }\end{array}$} & \multicolumn{3}{|c|}{$\begin{array}{c}35-44 \text { years } \\
(\mathrm{N}=840)\end{array}$} & \multicolumn{3}{|c|}{$\begin{array}{c}45-60 \text { years } \\
(\mathrm{N}=951)\end{array}$} & \multicolumn{3}{|c|}{ Overall } & \multicolumn{3}{|c|}{$35-44$ years } & \multicolumn{3}{|c|}{$45-60$ years } \\
\hline & $\beta$ & SE & P-value & $B$ & SE & P-value & $\beta$ & SE & P-value & $B$ & SE & P-value & $B$ & SE & $P$-value & $\beta$ & SE $\quad \mathrm{F}$ & $\mathrm{P}$-value \\
\hline Day work & 0 & & & 0 & & & 0 & & & 0 & & & 0 & & & 0 & & \\
\hline 3-shift & 0.337 & 0.048 & $<0.0001$ & 0.361 & 0.068 & $<0.0001$ & 0.367 & 0.075 & $<0.0001$ & 0.157 & 0.052 & 0.002 & 0.190 & 0.072 & 0.009 & 0.182 & 0.081 & 10.024 \\
\hline 5-shift & 0.163 & 0.046 & $<0.0001$ & 0.229 & 0.068 & 0.001 & 0.123 & 0.067 & 0.067 & 0.121 & 0.050 & 0.015 & 0.212 & 0.069 & 0.002 & 0.033 & 0.078 & 80.678 \\
\hline
\end{tabular}

a Partially adjusted model: $B$ corrected for long-term illness, taking care of children living at home and taking care of a chronic ill person living at home. In the healthcare results, this model is also corrected for educational level and gender.

${ }^{b}$ Fully adjusted model: $\beta$ additionally corrected for psychological job demands, decision latitude, emotionally and physically demanding work.

Table 2b. Overall and age-stratified regression coefficients and standard errors for continuous need for recovery scores according to work schedule among the industry and healthcare sector (TO-T8) [SE=standard error.]

\begin{tabular}{|c|c|c|c|c|c|c|c|c|c|c|c|c|c|c|c|c|c|c|}
\hline \multirow[t]{3}{*}{ Healthcare } & \multicolumn{9}{|c|}{ Model $1^{\text {a }}$} & \multicolumn{9}{|c|}{ Model $2^{b}$} \\
\hline & \multicolumn{3}{|c|}{$\begin{array}{c}\mathrm{N}=780 \\
\text { (595 women) }\end{array}$} & \multicolumn{3}{|c|}{$\begin{array}{c}35-44 \text { years } \\
(\mathrm{N}=483)\end{array}$} & \multicolumn{3}{|c|}{$\begin{array}{c}45-60 \text { years } \\
(\mathrm{N}=297)\end{array}$} & \multicolumn{3}{|c|}{ Overall } & \multicolumn{3}{|c|}{$35-44$ years } & \multicolumn{3}{|c|}{$45-60$ years } \\
\hline & $B$ & SE & P-value & $B$ & SE & P-value & $B$ & SE & P-value & $B$ & SE & P-value & $B$ & SE & P-value & $B$ & SE & P-value \\
\hline Day work & 0 & . & . & 0 & . & . & 0 & . & . & 0 & & . & 0 & . & . & 0 & . & \\
\hline $\begin{array}{l}\text { Irregular } \\
\text { shift }\end{array}$ & 0.140 & 0.063 & 0.027 & 0.013 & 0.077 & 0.863 & 0.418 & 0.108 & $<0.0001$ & 0.058 & 0.072 & 0.419 & -0.052 & 0.086 & 0.539 & 0.311 & 0.125 & 0.013 \\
\hline
\end{tabular}

Table 3. Overall and age-stratified hazard ratios (HR) and $95 \%$ confidence intervals $(95 \% \mathrm{Cl})$ for need for recovery caseness according to work schedule among the industry and healthcare sector (TO-T9)

\begin{tabular}{|c|c|c|c|c|c|c|c|c|c|c|c|c|c|c|c|}
\hline & \multicolumn{9}{|c|}{ Model $1^{\text {a }}$} & \multicolumn{6}{|c|}{ Model $2{ }^{b}$} \\
\hline & \multicolumn{3}{|c|}{ Overall } & \multicolumn{3}{|c|}{$35-44$ years } & \multicolumn{3}{|c|}{$45-60$ years } & \multicolumn{2}{|c|}{ Overall } & \multicolumn{2}{|c|}{$35-44$ years } & \multicolumn{2}{|c|}{$45-60$ years } \\
\hline & $\mathrm{N}$ & $\mathrm{HR}$ & $95 \% \mathrm{Cl}$ & $\mathrm{N}$ & $\mathrm{HR}$ & $95 \% \mathrm{Cl}$ & $\mathrm{N}$ & $\mathrm{HR}$ & $95 \% \mathrm{Cl}$ & $\mathrm{HR}$ & $95 \% \mathrm{Cl}$ & $\mathrm{HR}$ & $95 \% \mathrm{Cl}$ & $\mathrm{HR}$ & $95 \% \mathrm{Cl}$ \\
\hline Industry & 1408 & & & 659 & & & 749 & & & & & & & & \\
\hline Day work & & 1 & & & 1 & & & 1 & & 1 & & 1 & & 1 & \\
\hline 3-shift & & 1.37 & $1.04-1.80$ & & 1.28 & $0.88-1.87$ & & 1.44 & $0.93-2.25$ & 1.13 & $0.84-1.51$ & 1.05 & $0.70-1.58$ & 1.27 & $0.80-2.00$ \\
\hline 5-shift & & 1.03 & $0.80-1.32$ & & 1.08 & $0.74-1.56$ & & 0.97 & $0.67-1.40$ & 0.94 & $0.72-1.22$ & 1.02 & $0.69-1.49$ & 0.84 & $0.57-1.23$ \\
\hline Healthcare & & & & 414 & & & 235 & & & & & & & & \\
\hline Men & 156 & & & & & & & & & & & & & & \\
\hline Women & 493 & & & & & & & & & & & & & & \\
\hline Day work & & 1 & & & 1 & & & 1 & & 1 & & 1 & & 1 & \\
\hline $\begin{array}{l}\text { Irregular } \\
\text { shift }\end{array}$ & & 1.03 & $0.73-1.45$ & & 0.89 & $0.59-1.33$ & & 1.44 & $0.73-2.87$ & 0.88 & $0.61-1.27$ & 0.78 & $0.51-1.21$ & 1.18 & $0.55-2.54$ \\
\hline
\end{tabular}

ciations of work schedule with the continuous mean score and caseness of NFR were investigated. As the number of transitions from shift to day work was relatively low, all industry employees involved in any shift type at T0 were included $(\mathrm{N}=2048)$. The continuous score resulted in an OR 1.008 (95\% CI 1.004-1.011) indicating that employees with higher NFR scores on the $0-100$ scale were more likely to undergo a transition from shift to day work. For NFR caseness, an OR 1.61 (95\% CI 1.26-2.06) was found, indicating that employees with elevated NFR had higher odds of changing from shift to day work as compared to employees without elevated NFR. Among shift workers aged 45 years or older, these ORs were 1.007 (95\% CI 1.001-1.013) and $1.50(95 \%$ CI 0.94-2.40) respectively. 
Table 4. Overall and age-stratified hazard ratios (HR) and $95 \%$ confidence intervals ( $95 \% \mathrm{CI})$ for disability during follow-up according to work schedule among the industry and healthcare sector (TO-T9)

\begin{tabular}{|c|c|c|c|c|c|c|c|c|c|}
\hline & \multicolumn{3}{|c|}{ Overall } & \multicolumn{3}{|c|}{$35-44$ years } & \multicolumn{3}{|c|}{$45-60$ years } \\
\hline & $\mathrm{N}$ & $\mathrm{HR}$ & $95 \% \mathrm{Cl}$ & $\mathrm{N}$ & $\mathrm{HR}$ & $95 \% \mathrm{Cl}$ & $\mathrm{N}$ & $\mathrm{HR}$ & $95 \% \mathrm{Cl}$ \\
\hline Industry & & & & 872 & & & 1005 & & \\
\hline Men & 1877 & & & & & & & & \\
\hline Day work & & 1 & & & 1 & & & 1 & \\
\hline 3-shift & & 2.03 & $1.46-2.83$ & & 1.92 & $1.15-3.22$ & & 2.49 & $1.56-3.97$ \\
\hline 5-shift & & 1.74 & $1.28-2.36$ & & 1.77 & $1.07-2.93$ & & 1.80 & $1.20-2.70$ \\
\hline Healthcare & & & & 501 & & & 317 & & \\
\hline Men & 194 & & & & & & & & \\
\hline Women & 624 & & & & & & & & \\
\hline Day work & & 1 & & & 1 & & & 1 & \\
\hline Irregular work & & 1.35 & $0.93-1.95$ & & 1.09 & $0.66-1.79$ & & 2.04 & $1.18-3.54$ \\
\hline
\end{tabular}

Table 5. Age-stratified need for recovery mean score for different durations of exposure to shift work.

\begin{tabular}{|c|c|c|c|c|c|c|}
\hline \multirow{3}{*}{$\begin{array}{l}\text { Duration } \\
\text { of exposure }\end{array}$} & \multicolumn{6}{|c|}{ Industry } \\
\hline & \multicolumn{2}{|c|}{$\begin{array}{l}35-39 \text { years } \\
(\mathrm{N}=148)\end{array}$} & \multicolumn{2}{|c|}{$\begin{array}{c}\text { 40-44 years } \\
(\mathrm{N}=110)\end{array}$} & \multicolumn{2}{|c|}{$\begin{array}{c}45-50 \text { years } \\
(\mathrm{N}=111)\end{array}$} \\
\hline & Mean & P-value & Mean & P-value & Mean & P-value \\
\hline All & & $<0.0001^{a}$ & & $<0.0001$ & & $<0.0001^{b}$ \\
\hline $\begin{array}{l}\text { Short } \\
\text { ( } \leq 10 \text { years) }\end{array}$ & 44.61 & & 32.40 & & 42.24 & \\
\hline $\begin{array}{l}\text { Medium } \\
\text { (11-20 years ) }\end{array}$ & 25.19 & & 37.65 & & 46.85 & \\
\hline $\begin{array}{l}\text { Long } \\
\text { (>20 years) }\end{array}$ & 22.75 & & 43.90 & & 37.40 & \\
\hline
\end{tabular}

a Except medium versus long duration: $P=0.033$.

${ }^{\mathrm{b}}$ Except short versus long duration: $\mathrm{P}=0.015$.

\section{Discussion}

\section{Key findings}

This study aimed to investigate whether shift workers differ in terms of their NFR, future disability and retirement intentions compared to day workers over the span of the work career. Additionally, we investigated whether exposure duration to shift work was associated with NFR and whether we could illustrate a possible healthy worker effect.

The cross-sectional results demonstrated that in general, NFR levels at baseline were higher among shift workers as compared to day workers who are employed in the same sector (data for continuous NFR scores not shown). Few studies have investigated the association between shift work and NFR: however, in a different subsample of the Maastricht Cohort Study (18), a similar pattern was observed. Furthermore, all workers aged 45-60 years reported higher levels of NFR when compared to younger workers employed in the same shift type, implying that older employees might be less tolerant to the adverse effects of shift work, which is in line with an earlier study (13). This study demonstrated that also longitudinally both three- and five-shift industry workers reported higher NFR scores, as compared to industry day workers. After age stratification, this finding was no longer observed among the oldest age category of five-shift workers: it is likely that the healthy worker effect has contributed to this finding. The age-stratified results demonstrated that among the older irregular healthcare shift workers, significant higher NFR scores were observed as compared to older healthcare day workers, indicating that these shift workers might become more prone to the biological and social mechanisms related to shift work at an older age, and that the healthy worker effect has not (yet) taken place among these employees.

When investigating the risk of becoming a NFR case during the follow-up, only 3 -shift workers had a higher risk of becoming a NFR case as compared to industry day workers, a risk no longer present after age stratification. This study adds that NFR scores among many industry shift workers are higher over time as compared to day workers in that sector, but that the risk of becoming a NFR case is less present among industry shift workers. An explanation could be that shift workers have higher levels of NFR compared to day workers, but possibly do not have a high enough NFR level to become a case of elevated NFR, or that those shift workers who reach a NFR level high enough to become a case will, eg, change to day work jobs. To investigate whether long-term adverse effects of shift work were present, future disability was investigated. In line with earlier studies $(2,24)$, results showed that industry shift workers had a higher risk to become disabled during the follow-up as compared to industry day workers. Among healthcare workers, shift work was found to be a risk factor for future disability among the 45-60 age category. Several pathways could possibly explain this association between shift work and future disability, as shift work is negatively associated with both physical and mental health (19) and shift workers have a higher risk of occupational injury and illness as compared to day workers (32). 
Based on these adverse outcomes, it was also expected that shift workers would have stronger intentions to retire early as compared to day workers. However, this was not observed in this study. The healthy worker effect, practical limitations (retirement intentions were assessed at a later follow-up wave and among a smaller population) and the cross-sectional nature of this analysis could be possible explanations for the lack of this finding.

The actual direction and size of healthy worker effects have rarely been studied, therefore the present study aimed to investigate this effect explicitly among industry employees. Shift workers who quit shift work, reported higher NFR scores prior to the transition to day work, as compared to those who continuously performed shift work. Also, employees who were unable to perform shift work at an older age might already have left the labor force. Many studies presume the presence of this effect, whereas this study explicitly demonstrated that secondary selection processes took place in a sample of industry shift workers.

Furthermore, by investigating narrow age categories, the effect of exposure duration to shift work on NFR could be disentangled from the concept of age and results demonstrated that different lengths of exposure duration were associated with different NFR levels among industry shift workers. In the 35-39 age category, the highest NFR levels were found among employees with a short exposure duration to shift work and the lowest NFR levels among those with a long exposure duration. This might imply that it takes time for employees to adapt to the performance of shift work or that employees unable to adapt to shift work will make a transition, eg, to a day work job, supporting our conclusions on secondary selection processes which have taken place. Among employees aged 45-50 years, those with the longest exposure duration reported a lower NFR level, compared to those with a short or medium duration, possibly implying a healthy work effect within this age category.

All in all, the findings in this study might be an underestimation of the true results since secondary selection processes seem to have taken place. This might also partially explain why differences in NFR were observed among different age categories. Different shift work types and populations were investigated in two economic sectors with different working conditions. Consequently, the results of the industry and healthcare sector are not directly comparable. It can be concluded that despite these differences, in both economic sectors shift work was associated with higher levels of NFR and a higher risk of disability, when compared to day workers employed in the same sector.

Within the industry sector, in which two different shift work schedules were investigated, results also demonstrated that these two shift work schedules were differently associated with outcomes. Here, adverse outcomes were more often observed among 3-shift workers as compared to 5-shift workers. Possibly, these differences can be explained by the differences in number of working hours per week, which in general are higher among 2-shift schedules compared to 5 -shift schedules. Other explanations could be found in the difference in speed of rotation of the shifts, as in this cohort, 3-shift workers change shifts weekly, which is often considered to be most disadvantageous (33). Also, 3-shift workers in this cohort were frequently involved in backward rotating shift schedules, which are considered to be more disadvantageous as compared to forward rotating shift schedules (34).

\section{Strengths and limitations}

Several strengths and limitations should be considered. A strength of this study was that the extensive cohort allowed for the investigation of both short- and longterm outcomes expected to be related to shift work. Also, the analyses investigating the association of exposure duration and NFR and the explicitly investigated healthy worker effect should be considered strengths, as most studies on shift work do not explicitly examine these effects. Nevertheless, as the large scope of outcomes required several techniques of data analysis, different inclusion- and exclusion criteria, age categories, and follow-up waves, this might hinder direct comparisons between the various findings. Although a large variety of possible confounders were taken into account in this study, residual confounding cannot be completely ruled out. Shift work is a broad, ambiguous concept and many studies have compared shift to day workers. However, in this study, it was possible to differentiate multiple types of shift work. This distinction is valuable, as results differ among the specific type of shifts, implying that shift workers should be considered a heterogeneous population. Descriptive analyses revealed that the proportion of shift workers who transited to day work jobs was small. Nevertheless, it should be noted that the allocation to a shift or day work job was based on the employees work schedule at $\mathrm{T} 0$, which possibly might have resulted in misclassification. Eg, employees who had already made a transition from shift to day work before $\mathrm{T} 0$ were considered to be day workers, which might imply that selection processes already had taken place and results therefore may have revealed a somewhat diminished contrast between shift and day workers. As mixed findings exist regarding the effects of shift work across the work career, this study stratified the results for multiple age categories, allowing for a comparison between younger and older shift workers employed in the same economic sector, which was possible due to a large sample size. Although the widespread definition of the WHO was used to define older workers, different cut-off points exist in the literature, and chronological age might not 
always be sufficient to operationalize the factor "age" in the work setting (35). Therefore, a suggestion for future studies could be to also consider other approaches of the concept of age, eg, perceived relative age (36). As the Maastricht Cohort Study included employees from 45 different companies, a sector-specific approach was feasible in this study. The value of this approach is that shift workers could be compared to day workers within the same sector, implying rather similar work contexts, and therefore a more homogenous population within a sector could be investigated. Within each sector, this study aimed to construct a homogenous study population for the comparison of shift and day workers by applying relevant inclusion- and exclusion criteria. Nevertheless, the descriptive results revealed that both industry and healthcare shift workers had a higher prevalence of emotionally and physically demanding work compared to day workers employed in the same sector. Also, industry shift workers reported low levels of decision latitude compared to industry day workers, which is all in line with earlier studies $(37,38)$. Even after excluding employees with a high educational level from the industry population, the distribution of educational level differed among shift and day workers. Therefore, it can be concluded that shift work not only involves a different timing of working hours as compared to day workers, but also implies differences in job tasks and content, even when being employed in the same economic sector. Consequently, overcorrection has possibly taken place in the partially and fully adjusted models. Overall, it is difficult to construct a homogenous population of shift and day workers. By comparing shift and day workers within the same economic sector, this study attempted to establish a homogenous population. However, a suggestion for future research would be to compare shift to day workers with the same job title, which could contribute to further homogeneity of study populations.

\section{Concluding remarks}

Different associations between shift work and adverse outcomes were observed over the course of the work career. Shift work was generally associated with higher levels of NFR and a higher risk of disability over time, in both economic sectors. However, shift work is a multifaceted concept and although we were able to distinguish between different types of shift work, the influence of specific characteristics of shift types should be investigated in further detail in upcoming studies, eg, by taking into account the direction of rotation of shift schedules: among 3-shift workers a forward direction of shift rotation was prospectively related to less psychological health-related problems, in terms of NFR and general health, compared to a backwards-rotating schedule (34).
As this study illustrated that shift work exposure duration was associated with NFR and the healthy worker effect was present, the findings might be an underestimation of the true results and might partially explain why different associations across the age categories are observed. As these effects have not often been studied, a suggestion for further research could be to investigate how these effects are related to different outcome measures. For example, whereas this study demonstrated the healthy worker effect in terms of secondary selection processes, also primary and tertiary selection processes might take place and should therefore be studied. This study adds that shift work is generally associated with higher levels of NFR and a higher risk of disability, but that among shift workers different subgroups exist as different types of shift work schedules are differently associated with outcomes within a specific economic sector. As shift work schedules allow for optimization, this indicates that preventive measures should be tailored for different types of shift work over the course of the work career. Implications of this study are that implementation of (the type of) shift work within companies should be carefully considered, and companies in which shift work is currently performed should monitor and evaluate the health and performance of all shift workers as high NFR and disability among shift workers might result in higher costs for both employers and society.

\section{Acknowledgements}

The authors thank Jos Slangen for his support in the data management. Instituut Gak (grant no. 2011-089 WR) and School CAPHRI (School for Public Health and Primary Care), Maastricht, the Netherlands, financially supported this study.

The authors declare no conflict of interest.

\section{References}

1. Wang X-S, Armstrong MEG, Cairns BJ, Key TJ, Travis RC Shift work and chronic disease: the epidemiological evidence. Occup Med. 2011;61(2):78-89. http://dx.doi.org/10.1093/ occmed/kqr001.

2. Vogel M, Braungardt T, Meyer W, Schneider W. The effects of shift work on physical and mental health. J Neural Transm. 2012;119(10):1121-32. http://dx.doi.org/10.1007/s00702012-0800-4.

3. Dutch Association of Industry-wide Pension Funds. The Dutch Pension System: an overview of the key aspects. [Internet] 2010. [cited 2014 July 21]. Available from: http:// www.pensioenfederatie.nl/services/publicaties/Pages/ 
The_Dutch_Pension_System_36.aspx.

4. Gander P, Signal L. Who is too old for shift work? Developing better criteria. Chronobiol Int. 2008;25(2\&3):199-213. http:// dx.doi.org/10.1080/07420520802077556.

5. Costa G. Shift work and occupational medicine: an overview. Occup Med. 2003;53(2):83-8. http://dx.doi.org/10.1093/ occmed/kqg045.

6. Bambra C, Whitehead M, Sowden A, Akers J, Petticrew M. The health effects of reorganizing shift work. Am J Prev Med. 2008;34(5):427-34. http://dx.doi.org/10.1016/j. amepre.2007.12.023.

7. Jansen NWH, van Amelsvoort LGPM, Kristensen TS, van den Brandt PA, Kant IJ. Work schedules and fatigue: a prospective cohort study. Occup Environ Med. 2003;60(Suppl I):47-53. http://dx.doi.org/10.1136/oem.60.suppl_1.i47.

8. Harrington JM. Health effects of shift work and extended hours of work. Occup Environ Med. 2001;(58):68-72. http://dx.doi. org/10.1136/oem.58.1.68.

9. Bara A-C, Arber S. Working shifts and mental health -findings from the British Household Panel Survey (1995-2005). Scand J Work Environ Health. 2009;35(5):361-7. http://dx.doi. org/10.5271/sjweh.1344.

10. Driesen K, Jansen NWH, van Amelsvoort LGPM, Kant I. The mutual relationship between shift work and depressive complaints - a prospective cohort study. Scand J Work Environ Health. 2011;37(5):402-10. http://dx.doi.org/10.5271/ sjweh.3158.

11. Arendt J. Shift work: coping with the biological clock. Occup Med. 2010;60(1):10-20. http://dx.doi.org/10.1093/occmed/ kqp162.

12. Blok MM, de Looze MP. What is the evidence for less shift work tolerance in older workers? Ergonomics. 2011;54(3):221-32. http://dx.doi.org/10.1080/00140139.20 10.548876

13. Costa G, Di Milia L. Aging and shift work: a complex problem to face. Chronobiol Int. 2008;25(2\&3):165-81. http://dx.doi. org/10.1080/07420520802103410.

14. Saksvik IB, Bjorvatn B, Hetland H, Sandal GM, Pallesen S. Individual differences in tolerance to shift work - A systematic review. Sleep Med Rev. 2011;15(4):221-35. http://dx.doi. org/10.1016/j.smrv.2010.07.002.

15. Van Veldhoven M, Meijman TF. The measurement of psychosocial job demands with a questionnaire. Amsterdam: NIA; 1994

16. Van Veldhoven M. Need for recovery after work. An overview of construct, measurement and research. In: Houdmont J, Leka S, editors. Occupational Health Psychology: European perspectives on research, education and practice. Nottingham: Nottingham University Press; 2008. p. 1-25.

17. Jansen NW, Kant IJ, van den Brandt PA. Need for recovery in the working population: description and associations with fatigue and psychological distress. Int $\mathbf{J}$ Behav Med. 2002;9(4):322-40. http://dx.doi.org/10.1207/ S15327558IJBM0904_03.

18. Jansen NWH, Kant I, van Amelsvoort LGPM, Nijhuis FJN, van den Brandt PA. Need for recovery from work: evaluating short-term effects of working hours, patterns and schedules. Ergonomics. 2003;46(7):664-80. http://dx.doi.org/10.1080/ 0014013031000085662 .

19. De Croon EM, Sluiter JK, Frings-Dresen MH. Need for recovery after work predicts sickness absence: a 2-year prospective cohort study in truck drivers. J Psychosom Res. 2003;55:331-9. http://dx.doi.org/10.1016/S00223999(02)00630-X.

20. Merkus SL, van Drongelen A, Holte KA, Libriola M, Lund $\mathrm{T}$, van Mechelen $\mathrm{W}$, et al. The association between shift work and sick leave: a systematic review. Occup Environ Med. 2012;69(10):701-12. http://dx.doi.org/10.1136/ oemed-2011-100488.

21. Kubicek B, Korunka C, Hoonakker P, Raymo JM. Work and family characteristics as predictors of early retirement in married men and women. Res Aging. 2010;32(4):467-98. http://dx.doi.org/10.1177/0164027510364120.

22. Neophytou AM, Picciotto S, Hart JE, Garshick E, Eisen EA, Laden F. A structural approach to address the healthy-worker survivor effect in occupational cohorts: an application in the trucking industry cohort. Occup Environ Med. 2014;71:442-7. http://dx.doi.org/10.1136/oemed-2013-102017.

23. Bøggild H, Knutsson A. Shift work, risk factors and cardiovascular disease. Scand J Work Environ Health. 1999;25(2):85-99. http://dx.doi.org/10.5271/sjweh.410.

24. Waage S, Pallesen S, Moen BE, Bjorvatn B. Shift work and age in the offshore petroleum industry. Int Marit Health. 2010;61(4):251-7.

25. World Health Organization. Aging and working capacity: report of a WHO study group. Geneva: World Health Organization; 1993.

26. Van Veldhoven M, Broersen S. Measurement quality and validity of the "need forrecovery" scale. Occup Environ Med. 2003;60(1):i3-i9. http://dx.doi.org/10.1136/oem.60.suppl_1. i3.

27. Broersen JPJ, Fortuin RJ, Dijkstra M, van Veldhoven M, Prins J. Monitor Arboconvenanten: kengetallen en grenswaarden [Monitor working conditions agreements: indicators and cutoffs]. Tijdschrift voor Bedrijfs- en Verzekeringsgeneeskunde. 2004;(12):100-4.

28. Harkonmäki K, Rahkonen O, Martikainen P, Silventoinen K, Lahelma E. Associations of SF-36 mental health functioning and work and family related factors with intentions to retire early among employees. Occup Environ Med. 2006;63(8):558 63. http://dx.doi.org/10.1136/oem.2005.022293.

29. Houtman I. Reliability and validity of the Dutch version of the Karasek Job Content Questionnaire. Presented at: NIOSH/ APA conference on Stress, Work, and Health, 1995 Sept 14-16; Washington DC. USA.

30. Karasek R. The Job Content Questionnaire and User's Guide (version 1.1). Department of Industrial and Systems Engineering, Los Angeles: University of Southern California: 1985.

31. Gründemann RWM, Smulders PWG, de Winter CR. 
Handleiding Vragenlijst Arbeid en Gezondheid [Manual, Questionnaire on work and health]. Lisse: Swets \& Zeitlinger; 1993.

32. Dembe AE, Erickson JB, Delbos RG, Banks SM. Nonstandard shift schedules and the risk of job-related injuries. Scand J Work Environ Health. 2006;32(3):232-40. http://dx.doi. org/10.5271/sjweh.1004.

33. Knauth P. Designing better shift systems. Appl Ergon. 1996;27(1):39-44. http://dx.doi.org/10.1016/00036870(95)00044-5.

34. Van Amelsvoort LGPM, Jansen NWH, Swaen GMH, van den Brandt PA, Kant I. Direction of shift rotation among three-shift workers in relation to psychological health and work-family conflict. Scand J Work Environ Health. 2004;30(2):149-56. http://dx.doi.org/10.5271/sjweh.772.
35. Sterns HL, Alexander RA. Industrial gerontology: the aging individual and work. Annu Rev Gerontol Geriatr. 1987;7:24364.

36. Kooij D, de Lange A, Jansen P, Dikkers J. Older workers' motivation to continue to work: five meanings of age. J Manag Psychol. 2008;23(4):364-94. http://dx.doi. org/10.1108/02683940810869015.

37. Tenkanen L, Sjöblom T, Kalimo R, Alikoski T, Härmä M. Shift work, occupation and coronary heart disease over 6 years of follow-up in the Helsinki Heart Study. Scand J Work Environ Health. 1997;23(4):257-65. http://dx.doi.org/10.5271/ sjweh.218.

38. Härmä M. Workhours in relation to work stress, recovery and health. Scand J Work Environ Health. 2006;32(6):502-14. http://dx.doi.org/10.5271/sjweh.1055.

Received for publication: 12 September 2014 